Gut, 1987, 28, 1433-1438

\title{
Segmental necrotising enterocolitis: pathological and clinical features of 22 cases in Bangladesh
}

\author{
T BUTLER, B DAHMS, K LINDPAINTNER, M ISLAM, M A K AZAD, \\ AND P ANTON
}

From the International Centre for Diarrhoeal Disease Research, Bangladesh; and Departments of Medicine and Pathology, Case Western Reserve University, Cleveland, Ohio, USA

SUmmary To describe the pathology and clinical features of segmental necrotising enterocolitis (SNE) in children and adults, 22 diarrhoeal patients (median age two years, range two months to 50 years) in Bangladesh with this lesion detected at autopsy were examined and compared with two groups of diarrhoeal control patients. Gross pathology consisted of purplish or black mucosal or transmural discoloration with erosions or ulcerations in segments of the jejunum or ileum of 18 cases and of the colon alone in four cases. Two patients had intestinal perforations. Microscopically all specimens showed coagulation necrosis or haemorrhagic necrosis indicative of mucosal ischaemia. In 20 cases there was submucosal oedema and nine showed pneumatosis of the bowel. From 11, one or more of the invasive diarrhoeal pathogens Shigella, Campylobacter and Entamoeba histolytica were detected. From the comparison with controls significant associations were found for a long duration of diarrhoea, blood and mucus in stool, abdominal distension or tenderness, shock not attributable to hypovolaemia, septicaemia, and low concentration of serum protein $(\mathrm{p}<() \cdot() 5)$. These findings indicated that segmental necrotising enterocolitis develops sometimes as a fatal complication of prolonged diarrhoeal illnesses associated with shock and hypoproteinaemia and is caused by ischaemic injury to the intestinal mucosa.

Segmental necrotising enterocolitis (SNE) is an acute and often fatal complication of infectious diarrhoea, usually occurring in children in developing countries. This intestinal disease is a recently recognised entity, which has been reported as identical clinical syndromes or closely related variants under the names of 'segmental infarcts of the small intestine' and 'acute segmental ischaemic enteritis' in Thailand, ': 'acute segmental necrotising enteritis' and 'acute ischemic enteritis' in India, "necrotising enterocolitis' in Kuwait, "necrotising enteritis' in Sri Lanka," and 'enteritis necroticans' (pig-bel) in New Guinea. Although various aetiologic agents have been proposed for SNE, including round worms, ${ }^{4}$ the $\beta$-toxin of Clostridium perfringens, ${ }^{7}$ and sweet potato trypsin inhibitor." the pathogenesis of SNE remains unclear.

In an autopsy series of diarrhoeal patients recently done in Bangladesh." 22 patients with SNE were identified. We describe in this report for the first time

Address for correspondence: Dr T Butler, Dept of Internal Medicine, Texas Tech University Health Sciences Center. Lubbock, Texas 79430, USA.

Received for publication 9 April 1987 the findings of SNE among diarrhoea patients in Bangladesh and compare systematically the clinical features of patients with SNE to the features of similar groups of diarrhoeal patients who did not develop SNE.

\section{Methods}

PATIENTS

The International Centre for Diarrhoeal Disease Research in Dhaka, Bangladesh maintains a hospital for diarrhoeal diseases. Twenty two cases of SNE were identified among 140 autopsies on diarrhoeal patients carried out between May 1982 and May 1985. Autopsies were carried out as early as written informed consent could be obtained from families. Bodies were stored in a cold room before the autopsy. The time after death that autopsies were done ranged from one to 48 hours, with a median time of seven hours. In all cases the diagnosis of SNE was made at autopsy. All patients showed one or more discrete segments of the gastrointestinal tract with purple or black mucosal discolourations and 
erosions or ulcers recognised on gross examination. Microscopic examination used routinely formalin fixed and paraffin embedded sections stained with haematoxylin and eosin. All cases showed acute mucosal ischaemia with coagulation necrosis (ghost like villi) or haemorrhagic mucosal necrosis. Some inflammation was usually also present in the affected areas.

Clinical features of SNE cases were obtained by reviewing clinical records. To determine which features were significantly associated with SNE, retrospective case control analyses were performed. Two groups of diarrhoeal controls were selected for comparison. One group was 44 autopsy controls that were selected from the same series of autopsies by choosing two patients without SNE with serial autopsy numbers as close to each SNE case as possible. The other control group consisted of 44 surviving patients. Using a random number table to select serial hospital numbers, four controls were selected for each of the first 11 SNE cases, admitted during May 1982 to March 1983, from among surviving diarrhoeal patients admitted to the intensive care unit during the same two week period. Criteria for admission were signs of serious illness including respiratory distress, shock, altered mental status, seizures, and malnutrition. During the following period May 1983 to May 1985 an additional 11 cases of SNE were detected at autopsy. Chart review was then performed for cases and controls. Data collected included information concerning age, sex, history, physical examination, and laboratory data. Shock was defined as absent or thready radial pulse that persisted after intravenous infusion of isotonic fluid in an amount $\geq 5 \%$ of the body weight. Septicaemia was defined by positive antemortem blood cultures for pathogenic bacteria or positive postmortem blood cultures if taken less than six hours after death. Mean values were determined for both SNE cases and control groups and compared by Student's $t$ test. Frequencies were evaluated by the $\chi^{2}$ test or Fisher exact test.

\section{Results}

PATHOLOGIC FINDINGS IN SNE

On opening the abdominal cavity at autopsy one or more discrete segments of intestine were noted to be purple or black in colour. This was transmural and apparent on the serosal surface in 15 patients (Fig. $1)$; in the remaining seven the mucosa alone was involved. The small intestine, particularly distal jejunum and ileum, was the most common site of the segmental necrosis. In eight individuals, lesions were limited to small intestinal segments. Ten patients had involvement of both small intestine and colon. In only four patients was the colon alone involved. When the bowel was opened, the involved segments revealed deeply congested or haemorrhagic mucosa with superficial erosions or ulcerations. The length of involved segments was highly variable, some patients had patchy involvement with ischaemic areas only several centimetres long. In half the patients, one third or more of the length of the small intestine was congested and haemorrhagic. In one infant the entire small intestine was grossly purple. Two adult patients demonstrated one or more perforations through the involved segments (one small intestinal, one colonic).

Microscopically the lesions most closely resembled ischaemic bowel disease. Foci of mucosa in all cases showed coagulative necrosis, usually with ghost like villi devoid of distinct cellular outlines and nuclear detail (Fig. 2). In some cases only villus tips were involved; more advanced lesions showed complete mucosal and even submucosal extension. Haemorrhagic necrosis was also seen focally in all cases (Fig. 3 ) and is responsible for the dark red to black colour which allows for gross recognition of involved intestinal segments. Usually this coagulative and haemorrhagic necrosis was combined with inflammation (Fig. 2). In some cases where only the superficial mucosa showed coagulative necrosis, the inflammation was subjacent, with acute and chronic inflammatory cells filling the viable lamina propria. In cases with coagulative necrosis of the entire mucosa, the inflammation was apparent immediately adjacent. No bacteria were identified in these areas, even though they may have been present elsewhere in the gastrointestinal tract. Submucosal oedema was present in 20 of the cases, expanding this layer of the bowel to approximately two or three times normal thickness (Fig. 3). Nine patients demonstrated extensive pneumatosis intestinalis of the submucosa (Fig. 4). These submucosal gas bubbles were present in both small intestine and colon (small intestine alone in four patients, colon alone in one patient, both in four patients). The muscularis propria was intact except in two patients with transmural gangrene and intestinal perforation. Thrombi were not seen in any large blood vessels in the intestines except in one patient who had hyphae of mucormycosis invading blood vessels in necrotic lesions of the terminal ileum and ascending colon."' Rare capillary platelet fibrin thrombi were observed in the submucosa in areas of coagulative necrosis. These were secondary to the necrosis and not aetiologic.

PATHOLOGY OF OTHER TISSUES

Mesenteric lymph nodes were enlarged in 14 cases and showed increased numbers of polymorphonuclear leukocytes. In two cases the lymph nodes showed erythrophagocytosis. The livers of 18 


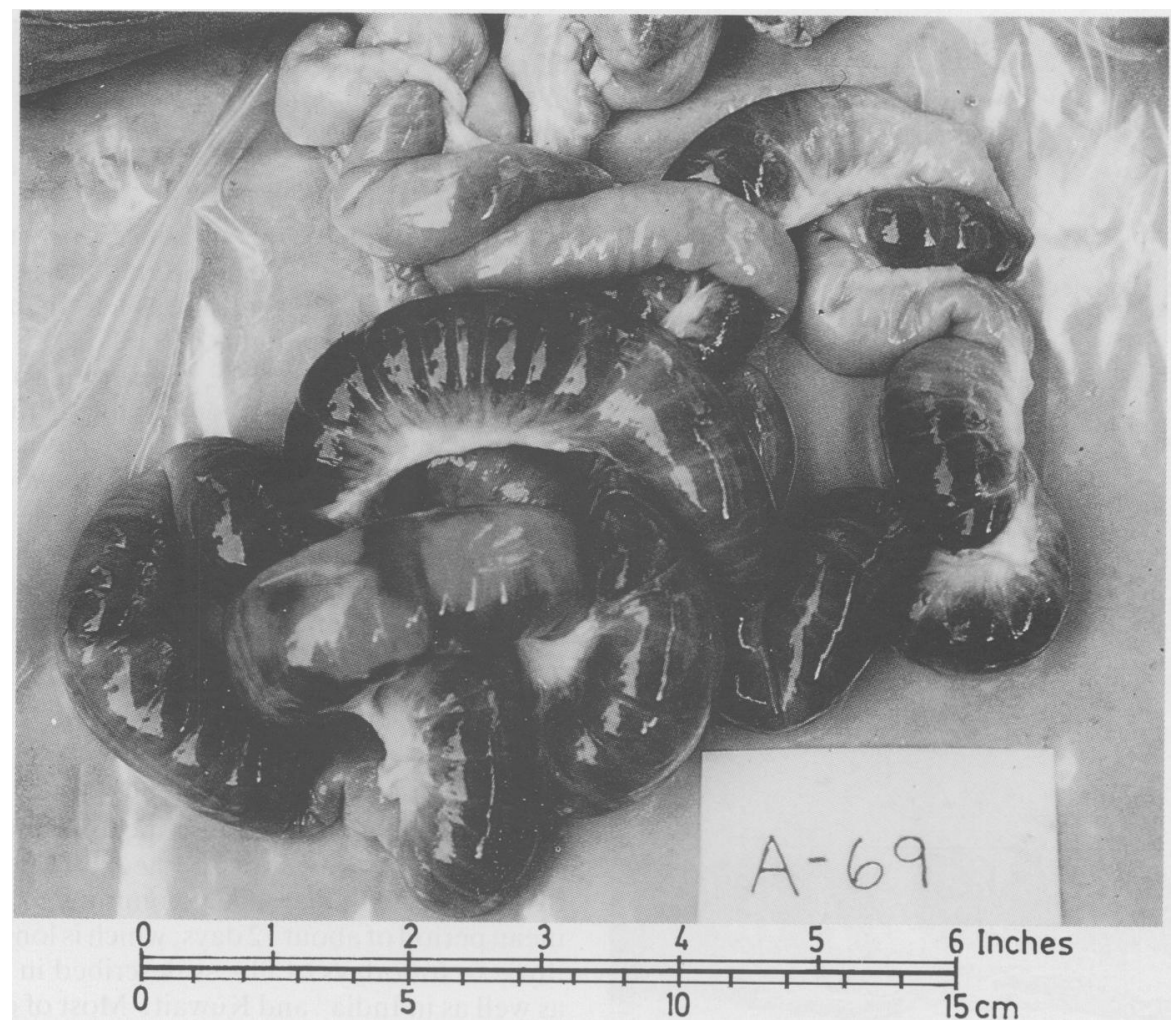

Fig. 1 Gross photograph of segmental necrotising enterocolitis shows distension and black discolouration of long and short segments of small intestine. Intervening lighter areas are spared.

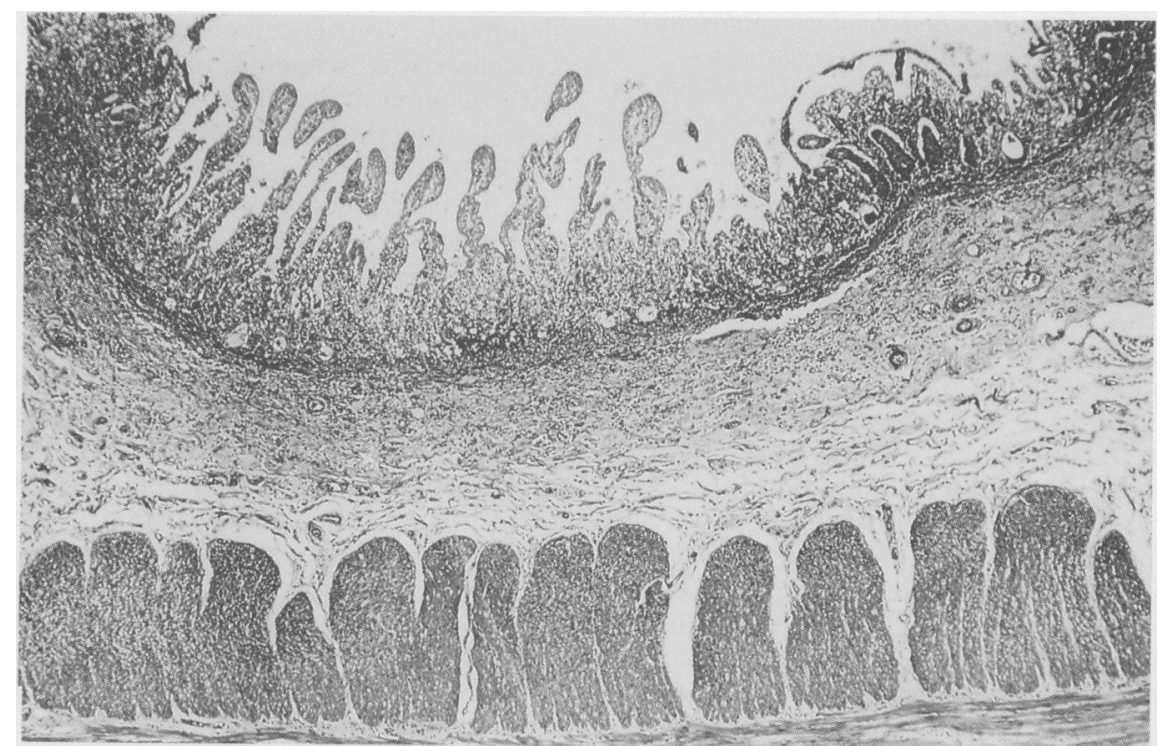

Fig. 2 Coagulative necrosis of small intestinal mucosa, seen at the centre is characterised by ghost like villi devoid of cellular detail. Also note superficial epithelium lifting off mucosal surface to the right. (Haematoxylin and eosin.) 


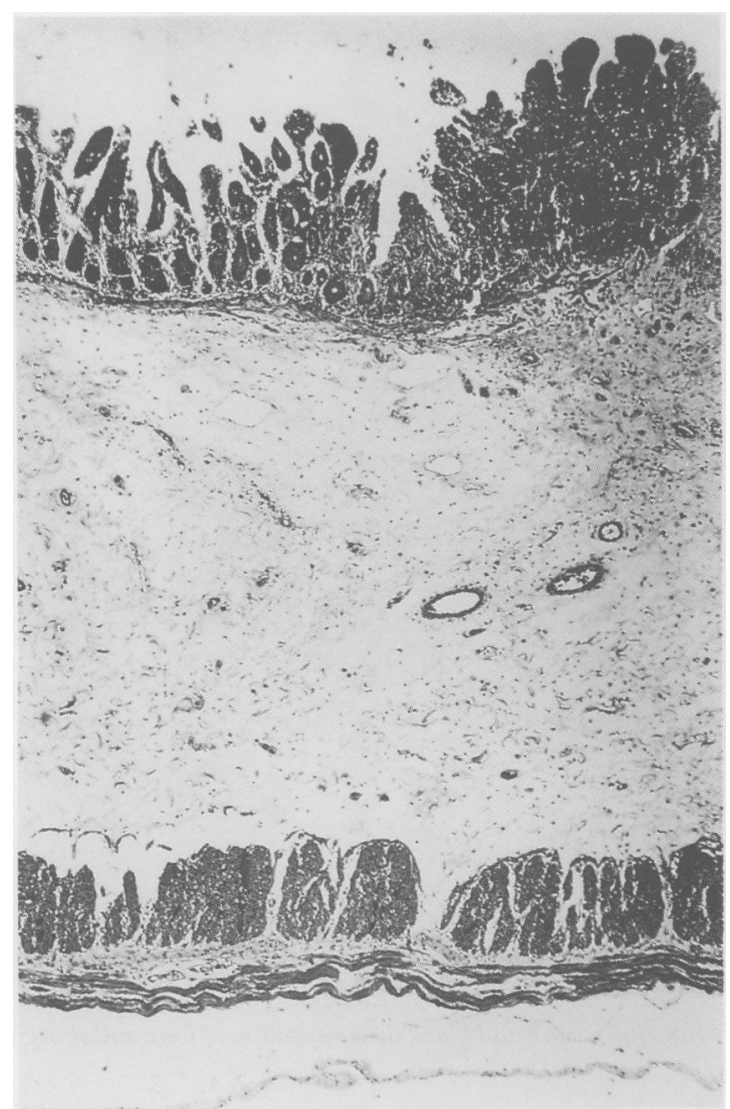

Fig. 3 Haemorrhagic mucosal necrosis is seen at the upper right. Cellular detail of surface and glandular epithelium is effaced, and lamina propria is filled with erythrocytes, imparting a dark colour to the affected area. The submucosa shows pronounced oedema. The muscularis layers are not involved. (Haematoxylin and eosin.)

patients showed fatty infiltration. The degree of fatty infiltration was marked in 10 cases, moderate in six cases, and mild in two cases.

CIINICAI. AND LABORATORY FINDINGS

The median age of the SNE cases was two years (range two months to 50 years) and there were more female than male patients (Table). Eleven patients were hospitalised during the summer months July to September. Segmental necrotising enterocolitis patients reported a mean duration of diarrhoea of 11.9 days and were hospitalised for a mean of three days before death. Most of the patients with SNE had presented with blood and mucus in stool with vomiting and signs of dehydration. Most of them developed abdominal distension or tenderness and shock not attributed to saline depletion. Fever $\left(\mathrm{T} \geq 37 \cdot 8^{\circ} \mathrm{C}\right.$ on the day of admission) was detected in nine of the SNE patients. One or more of the invasive diarrheal pathogens Shigella sp, Entamoeba histolytica, and Campylobacter sp were detected in 11 cases. Cholera was diagnosed in none of the SNE cases. Septicaemia was diagnosed in eight cases $(36 \%)$. The mean white blood cell count was $17,300 / \mathrm{mm}^{3}$ and the mean concentration of serum protein was $4.5 \mathrm{~g} / 100 \mathrm{ml}$. Metabolic acidosis with total serum $\mathrm{CO}_{2}$ concentrations less than $20 \mathrm{mmol} / \mathrm{l}$ was detected in 16 patients with SNE.

Comparison of the SNE cases with autopsy diarrhoeal controls revealed comparable features except for a greater frequency of blood in stool of the SNE cases (Table) $(\mathrm{p}<0 \cdot 05)$. On the other hand, comparison of SNE cases with surviving diarrhoeal controls, showed that SNE cases had a longer mean duration of diarrhoea before admission, greater frequencies of blood and mucus in stool, abdominal distension or tenderness, shock, and septicaemia, and a lower mean concentration of serum protein $(p<0 \cdot(05)$.

\section{Discussion}

Our patients with SNE were mostly children over a wide range of ages after the neonatal period. They had been ill with diarrhoea, vomiting and fever for a mean period of about 12 days, which is longer than the three to four days of illness described in Thailand, '? as well as in India" and Kuwait. ${ }^{5}$ Most of our patients presented with dysenteric diarrhoea, in contrast with the patients in Thailand who had watery diarrhoea. ' The portion of the bowel most commonly affected by segmental necrosis was the jejunum, followed by the ileum and colon. The microscopic pathology found in our cases was ischaemic or haemorrhagic necrosis, with oedema and without significant thrombosis of vessels, affecting the musoca and, sometimes, the full thickness of the intestinal wall and is typical of the disease described in other countries. ${ }^{1-n}$ Other variable features, including acute inflammation of the bowel, pneumatosis, and perforation, were sometimes present in our cases. None of our patients showed vasculitis or fibrinoid necrosis of intestinal blood vessels, as was described in Sri Lanka."

The aetiology of SNE is not known. Headington et $a l^{\prime}$ who described the clinical and pathologic findings of this condition in 1967, regarded it as segmental infarction. Most of the patients reported from Thailand' ' were not in shock at presentation and had no vessel occlusions or other discernible vascular lesions to explain the pathologic resemblance to ischemic bowel disease. There is a striking similarity of the pathology of SNE to neonatal necrotising enterocolitis (NEC), a disease largely of premature infants in intensive care units. ${ }^{12}$ The anatomic distribution of disease in NEC is most commonly the 


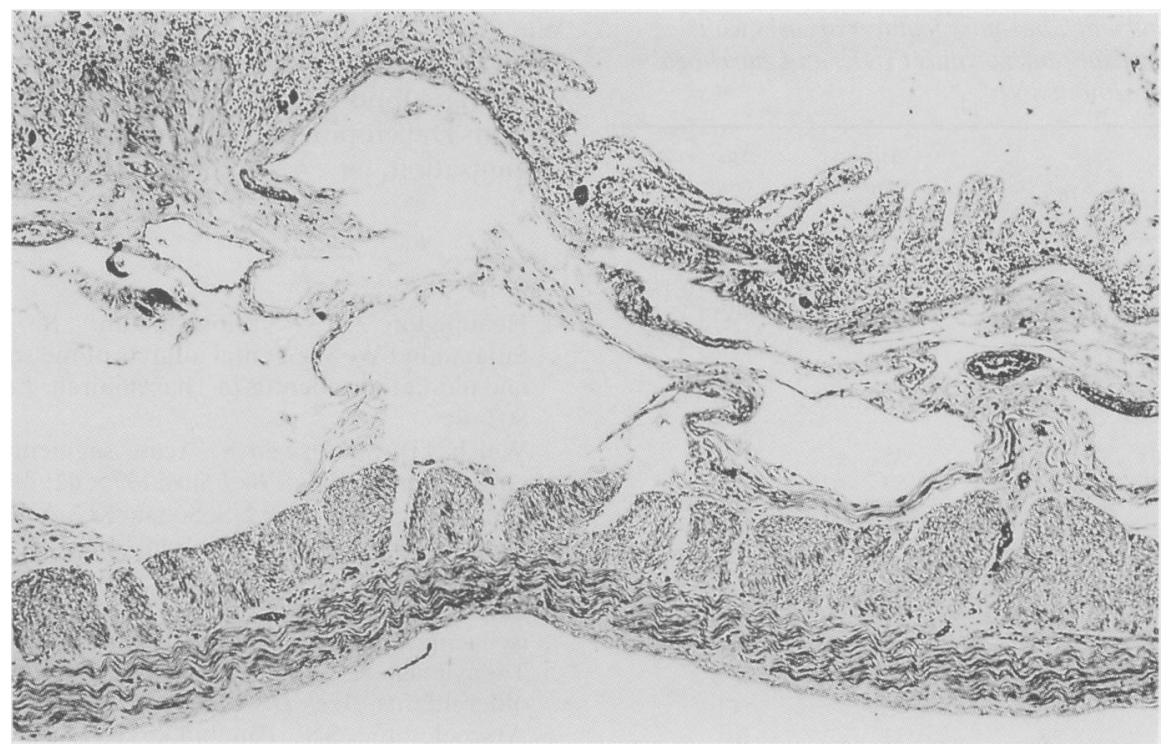

Fig. 4 Severe pneumatosis intestinalis is present in the submucosa of the small intestine. (Haematoxylin and eosin.)

terminal ileum and proximal colon, however in contrast the jejunum in SNE. The combination of ischaemic and inflammatory findings in NEC has led to the same aetiologic impasse in that disease: is it caused by ischaemia, infection, or a combination of factors?

Previous efforts to identify an aetiologic agent for SNE have not succeeded. Stool cultures for enteropathogens have shown negative results, except for one case of Salmonella. ${ }^{35}$ Although $C$ perfringens has been isolated from some patients, ${ }^{2}{ }^{2} 7$ the relevant toxin of this organism is the $\beta$-toxin of $C$ perfringens type $C$, which was identified in only nine of 54 isolates with toxin from patients in New Guinea.' Furthermore, the toxin is degraded by pancreatic proteases in the intestinal lumen. Half of our patients were infected with the invasive diarrhoeal pathogens Shigella sp, Entamoeba histolytica, and Campylobacter sp. The lesions of SNE were in the jejunum and those of shigellosis and amoebiasis in the colon, indicating that these primary intestinal infections did not directly cause the lesions of SNE.

Hypoproteinaemia probably contributed to hypoperfusion of the intestine in some of our patients by a reduction in plasma oncotic pressure. At serum concentrations of protein below $5 \mathrm{~g} / 100 \mathrm{ml}$, oedema of tissues occurs because fluid leaves the vessels under normal hydrostatic pressure in the arterial circulation and the diminished osmotic pressure in the venous side of the capillaries fails to draw tissue fluid back into the vessels. ${ }^{13}$ Oedema of the intestinal wall, present in our cases and reported in other studies of ischaemic enteritis, ${ }^{12 t}$ is consistent with this mechanism. Severe body burns and nontropical sprue, which cause large losses of plasma proteins, are also associated with necrosis of the intestinal mucosa. ${ }^{1+15}$ In the carcinoid syndrome and the acquired immunodeficiency syndrome, the presence of diarrhoea is associated with hypoalbuminaemia. ${ }^{16} 17$ Thus, hypoalbuminaemia could serve as a common stimulus for both diarrhoea and SNE through a deficit in plasma oncotic pressure. ${ }^{\text {Is }}$ The causes of hypoproteinaemia in our cases were not identified but probably include decreased intake of protein, protein losing enteropathy, malabsorption, and decreased albumin synthesis by the liver. ${ }^{16}$

Local factors in the intestine may also act to predispose the bowel to ischaemic injury. When the small intestine is stimulated by an enterotoxin to secrete fluid during diarrhoea, the intestine will require additional perfusion to obtain fluid for secretion as well as to maintain its oxygen supply and nutrients. Thus, the enterotoxin stimulated jejunum or ileum may become more vulnerable to ischaemic injury when there is not an ample reserve of perfusion pressure and volume. Most of our patients presented with arterial hypotension often caused by septicaemia, which produces a sympathetic nervous reflex leading to secretion of catecholamines. Catecholamines will act to redistribute blood flow away from the intestine, thus aggravating or provoking intestinal ischaemia. ${ }^{11}$ Another factor in the pathogenesis of SNE is autodigestion of the mucosa by pancreatic proteases, including trypsin, chymo- 
Table Clinical and laboratory features of cases with segmental necrotising enterocolitis (SNE) and diarrhoeal control groups without $S N E^{*}$

\begin{tabular}{|c|c|c|c|}
\hline & $\begin{array}{l}\text { SNE cases } \\
(n=22)\end{array}$ & $\begin{array}{l}\text { Autopsy } \\
\text { controls } \\
(n=44)\end{array}$ & $\begin{array}{l}\text { Surviving } \\
\text { controls } \\
(n=44)\end{array}$ \\
\hline \multicolumn{4}{|l|}{ Median age (range). } \\
\hline Males/females (n) & $8 / 14$ & $23 / 21$ & $30 / 14$ \\
\hline $\begin{array}{l}\text { Duration of diarrhoea } \\
\text { before admission, } \\
\text { dayst }\end{array}$ & $11 \cdot 9 \pm 9 \cdot 5$ & $14 \cdot 1 \pm 18 \cdot 1$ & $5 \cdot 2 \pm 6 \cdot 5$ \\
\hline \multicolumn{4}{|l|}{ Percent with: } \\
\hline Blood in stool $\$$ & 55 & 25 & 11 \\
\hline Mucus in stool§ & 77 & 55 & 32 \\
\hline Vomiting & 55 & 45 & 86 \\
\hline Dehydration & 59 & 73 & 52 \\
\hline $\begin{array}{l}\text { Abdominal } \\
\text { distension or } \\
\text { tenderness } \$\end{array}$ & 64 & 41 & 11 \\
\hline $\begin{array}{l}\text { Shock not attributed } \\
\text { to saline } \\
\text { depletion\$ }\end{array}$ & 55 & 45 & 11 \\
\hline Shigella $s p$ & 18 & 32 & 10 \\
\hline Entamoeba histolytica & 18 & 16 & 7 \\
\hline Campylobactersp & 18 & 17 & - \\
\hline Vibrio cholerae & 0 & 9 & 24 \\
\hline Septicacmia & 36 & 36 & 14 \\
\hline \multicolumn{4}{|c|}{ White blood cell count $/ \mathrm{mm}^{3}$} \\
\hline$\times 10(0)$ & $17 \cdot 3 \pm 9 \cdot 1$ & $17 \cdot 2 \pm 15 \cdot 4$ & $17 \cdot 6 \pm 15 \cdot 8$ \\
\hline $\begin{array}{l}\text { Total serum protein } \\
\mathrm{g} / 100 \mathrm{ml}+\end{array}$ & $4 \cdot 5 \pm 1 \cdot 1$ & $5 \cdot() \pm 1 \cdot 6$ & $6 \cdot 5 \pm 1 \cdot 6$ \\
\hline
\end{tabular}

${ }^{*}$ Values are expressed as means \pm SD or per cents. See Methods for description of control groups: $†$ Mean value of SNE cases significantly different from mean value of surviving controls by Student's $t$ test $(p<0 \cdot(0) 5)$; $\ddagger$ Frequency of SNE cases significantly greater than autopsy controls and surviving controls by $\chi^{2}$ test $(p<0 \cdot(05)$ : \$Frequency of SNE cases significantly greater than surviving controls by $\chi^{2}$ and Fisher's exact test $(\mathrm{p}<0 \cdot(05)$.

trypsin, and elastase. After the initial ischaemic injury, the brush border of the mucosa loses its glycoprotein protection against luminal enzymes..$^{20}$ This autodigestion is a later event in SNE and may explain the mucosal erosions and ulcers in our patients.

When clinical features of our patients with SNE were compared with autopsy controls, only blood in stools occurred more frequently in the SNE cases. On the other hand, when clinical features were compared with surviving diarrhoeal controls, the SNE cases showed a longer duration of diarrhoea, a lower serum protein concentration, and greater frequencies of blood and mucus in stool, abdominal distension or tenderness, septicaemia, and shock. This suggests that the clinical features of SNE are the same as those associated with a fatal outcome in the setting of diarrhoeal diseases. Thus, the emergence of SNE before death may be determined by crucial timing of an altered intestinal circulation in severely ill patients.
We thank M R Islam, Peter Speelman, and John Banwell for their assistance with the manuscript. This work was supported, in part, by a grant from United Nations Development Programme and World Health Organisation.

\section{References}

1 Headington JT, Sathornsumathi S, Simark S, Sujatanond $W$. Segmental infarcts of the small intestine and mesenteric adenitis in Thai children. Lancet 1967; i: 802-6.

2 Welch TP, Sumitswan S. Acute segmental ischaemic enteritis in Thailand. Br J Surg 1975; 62: 716-9.

3 Kalani BP, Shekhawat NS, Sogani KC. Acute segmental necrotizing enteritis in children. Am J Dis Child 1985; 139: $586-8$.

4 Gupta SD, D'Silva V, Salelkar AV, Reys M. Acute ischaemic enteritis in Goa. Br J Surg 1977; 64: 420-3.

5 Takayanagi K, Kapila L. Necrotising enterocolitis in older infants. Arch Dis Child 1981; 56: 468-71.

6 Arseculeratne SN, Panabokke RG. Navaratnam C. Pathogenesis of necrotising enteritis with special reference to intestinal hypersensitivity reactions. Gut 1980; 21: 265-78.

7 Murrell TGC, Roth L, Egerton J, Samels J, Walker PD. Pig-bel: enteritis necroticans. A study in diagnosis and management. Lancet 1966; i: 217-22.

8 Lawrence G, Walker PD. Pathogenesis of enteritis necroticans in Papua New Guinea. Lancet 1976; i: $125-6$.

9 Butler T, Islam M, Azad MAK, Islam MR, Speelman P. Causes of death in diarrheal diseases: an autopsy study of 140 patients in Bangladesh who died in hospital after rehydration. Bull Wld Hlth Org 1987; 65: 317-23.

10) Calle $\mathrm{S}$, Klatsky S. Intestinal phycomycosis (mucomycosis). Am J Clin Pathol 1966; 45: 264-72.

11 Zeissler J, Rassfeld-Sternberg L. Enteritis necroticans due to Clostridium welchii type F. Br Med J 1949; i: 267-9.

12 Kliegman RM, Fanaroff AA. Necrotizing enterocolitis. $N$ Engl J Med 1984; 310: 1093-103.

13 West ES, Todd WR. Textbook of Biochemistry. New York: The Macmillan Co, 1962: 522.

14 Patterson M, Rosenbaum HD. Enteritis necroticans. Gastroenterology 1952; 21: 110-8.

15 Bounous G. Acute necrosis of the intestinal mucosa. Gastroenterology 1982; 82: 1457-67.

16 Mariani G, Strober W, Keiser H, Waldman TA. Pathophysiology of hypoalbuminemia associated with carcinoid tumor. Cancer 1976; 38: 854-60).

17 Kotler DP, Gaetz HP, Lange M, Klein EB, Holt PR. Enteropathy associated with the acquired immunodeficiency syndrome. Ann Intern Med 1984; 101: 421-8.

18 Moss G. Plasma albumin and postoperative ileus. Surg Forum 1967; 18: 333-6.

19 Bynum TE, Jacobson ED. Nonocclusive intestinal ischemia. Arch Intern Med 1979; 139: 281-2.

20 Bounous G. Menard D, de Medicis E. Role of pancreatic proteases in the pathogenesis of ischemic enteropathy. Gastroenterology 1977; 73: 102-8. 